\title{
A new operator splitting algorithm for elastoviscoplastic flow problems
}

\author{
I. Cheddadi ${ }^{a, *}$, P. Saramito ${ }^{a, * *}$ \\ ${ }^{a}$ Lab. Jean Kuntzmann - CNRS and Université de Grenoble, F-38041 Grenoble, France
}

\begin{abstract}
This paper presents an efficient time-dependent decoupled approach for the numerical resolution of the highly nonlinear set of coupled partial differential equations appearing in elastoviscoplastic fluid flow problems. The two main nonlinear difficulties, the viscoplasticity and the viscoelasticity, are then solved separately. Numerical simulations suggest an optimal convergence rate with respect to the space discretization. Finally, numerical results compare well with experimental measurements on liquid foams in a complex geometry. Future works will explore flows of liquid foams for tridimensional geometries where experimental data are available and also compare to flows of others soft glassy materials such as carbopol solutions.
\end{abstract}

Keywords: liquid foams and emulsions, elastoviscoplasticity, yield stress, nonlinear viscoelasticity, numerical simulation

\section{Introduction}

Foams, gels, emulsions, polymer solutions, pastes [11] and even cell assemblies $[28,34]$ display both liquid and solid mechanical properties, being simultaneously Elastic (E), viscous (V), and plastic (P): at small deformation, such a material reversibly come back to its shape; at large deformation, it can be irreversibly sculpted and gets a new shape; under an increasing deformation rate, it irreversibly flows, with an increasing viscous stress.

In particular, liquid foams have been widely used to probe such properties as they allow detailed multiscale measurements; meanwhile, numerous continuous models of foam flows have been developed including for instance either a phenomenological scalar description $[25,22]$, or a complete tensorial description

\footnotetext{
*Present address: LJLL, UPMC, Boîte courrier 187, F-75252 Paris Cedex 05, France

${ }^{* *}$ Corresponding author

Email addresses: cheddadi@ann.jussieu.fr (I. Cheddadi), Pierre.Saramito@imag.fr (P. Saramito)
} 
of the elasticity [3] or plasticity [29] based on the micro-structure. In 2007, a new elastoviscoplastic (EVP) model was proposed [42, 43] that combined the Bingham [4] or Herschel-Bulkley [21] viscoplastic models with the Oldroyd [32] viscoelastic model into an EVP framework, suitable for flow simulations. This model was then shown to successfully reproduce some experimental measurements for impact of carbopol drops [27], Couette flows of liquid foams [7] or emulsions [6] and flows of liquid foams around an obstacle [5] (Fig. 1).

In the present paper, our aim is to present an efficient numerical algorithm based on mixed Finite Elements Methods for solving the highly nonlinear set of equations describing the EVP materials in complex geometries such as in Fig. 1. The formulation includes various boundary conditions, suitable for classical benchmarks for non-newtonian fluids such as constriction, or flow around an obstacle with arbitrary shape; in particular, its implementation allows slip boundary conditions, relevant for foam flows in channels, on arbitrarily shaped boundaries.

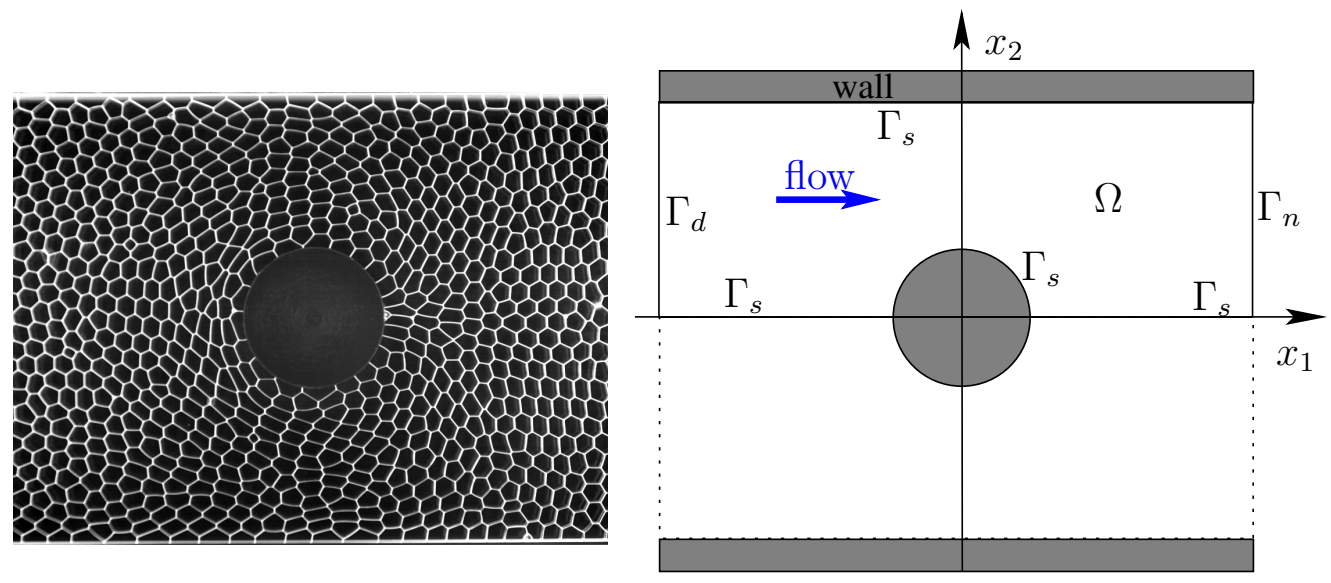

Figure 1: The bidimensional flow of a liquid foam around an obstacle: (left) photography from [12] ; (right) notations for the computation.

The algorithm extends a previous second order time-splitting algorithm introduced in the context of viscoelastic flow simulations [38, 41] and then applied to some nonlinear viscoelastic flow models $[40,44]$. A second order time-splitting algorithm, also called $\theta$-scheme, was originally introduced for Newtonian fluid dealing with inertia terms $[18,17]$ : the two main difficulties - incompressibility and the nonlinear inertia effects - were split and solved separately. The present algorithm deals with an additional highly nonlinear term introduced by the viscoplasticity in the EVP model: in a similar way, the two main difficulties viscoplasticity and viscoelasticity - are split and solved separately.

Our plan is as follows. In order to make this paper self-contained, section 2 recalls the model and the nonlinear set of equations to be solved. Section 3 
presents in details the numerical algorithm, together with time and space discretizations. It explains the algorithm we developed to deal with the strong non-linearities of EVP equations, and a well controlled space- and time discretization. Section 4 first shows a validation test on a Couette flow and then the results of simulations for a more complex flow around an obstacle.

\section{Constitutive equation for EVP fluids}

We briefly recall the constitutive equation for an EVP fluid proposed by [42]. The stress tensor is decomposed into three contributions:

$$
\boldsymbol{\sigma}=2 \eta_{1} D(\boldsymbol{v})+\boldsymbol{\tau}-p I,
$$

where $v$ is the velocity vector, $\boldsymbol{\tau}$ is the elastic stress tensor, $p$ is a scalar quantity akin to the pressure (see below in section 2), $\eta_{1}$ is a viscosity, and $D(\boldsymbol{v})=$ $1 / 2\left(\nabla \boldsymbol{v}+\nabla \boldsymbol{v}^{T}\right)$ is the deformation rate tensor, and $\nabla \boldsymbol{v}=\left(\partial v_{i} / \partial x_{j}\right)$ is the velocity gradient. The elastic stress $\boldsymbol{\tau}$ follows itself an evolution equation:

$$
\lambda \stackrel{\square}{\boldsymbol{\tau}}+\kappa\left(\left|\boldsymbol{\tau}_{d}\right|\right) \boldsymbol{\tau}-2 \eta_{2} D(\boldsymbol{v})=0,
$$

where $\lambda$ is the elastic relaxation time, $\eta_{2}$ is a viscosity, $\boldsymbol{\tau}_{d}=\boldsymbol{\tau}-1 / d \operatorname{tr}(\boldsymbol{\tau}) I$, $d=2$ or 3 , is the deviatoric part of $\boldsymbol{\tau},|\cdot|$ denotes the usual Euclidean matrix norm, $\kappa(\xi)=\max \left(0,1-\tau_{Y} / \xi\right), \forall \xi \in \mathbb{R}^{+}$describes plastic dissipation above the yield-stress $\tau_{Y}$, and

$$
\stackrel{\square}{\boldsymbol{\tau}}=\frac{\partial \boldsymbol{\tau}}{\partial t}+(\boldsymbol{v} \cdot \nabla) \boldsymbol{\tau}+\beta_{a}(\boldsymbol{\tau}, \nabla \boldsymbol{v})
$$

is an objective time derivative, where $\beta_{a}(.,$.$) denotes the following bilinear form$

$$
\beta_{a}(\boldsymbol{\tau}, \nabla \boldsymbol{v})=\boldsymbol{\tau} W(\boldsymbol{v})-W(\boldsymbol{v}) \boldsymbol{\tau}-a(D(\boldsymbol{v}) \boldsymbol{\tau}+\boldsymbol{\tau} D(\boldsymbol{v}))
$$

and $a \in[-1,1]$ is a parameter. Here, $W(\boldsymbol{v})=\left(\nabla \boldsymbol{v}-\nabla \boldsymbol{v}^{T}\right) / 2$ is the vorticity tensor.

Note that in the context of small strains, the elastic stress is proportional to the elastic deformation $\varepsilon^{e}=1 /(2 \mu) \tau$, where $\mu$ is the elastic modulus, allowing direct comparison with experiments where $\varepsilon^{e}$ can be measured, as in [5].

\section{Problem statement}

In order to obtain a closed set of equations, the constitutive equation is coupled with momentum and mass conservation equations for an incompressible material. The problem is stated in dimensionless form: let $L$ (resp. $V$ ) be some characteristic length (resp. velocity), then $W e=\lambda V / L, B i=\tau_{Y} L /\left(\eta_{2} V\right)$ and $R e=\rho V L /\left(\eta_{1}+\eta_{2}\right)$ are respectively the Weissenberg, Bingham and Reynolds 
dimensionless numbers [42], $\left.\left.\alpha=\eta_{2} /\left(\eta_{1}+\eta_{2}\right) \in\right] 0,1\right]$ is the so-called retardation parameter in the context of the Oldroyd model.

Let $\Omega \subset \mathbb{R}^{d}, d=2,3 T>0$, and $\Gamma_{d}, \Gamma_{n} \Gamma_{s}$ be a partition of its boundary $\partial \Omega$; the problem is expressed with dimensionless fields also denoted $(\boldsymbol{\tau}, \mathbf{v}, p)$ :

$(P)$ : find $\boldsymbol{\tau}, \boldsymbol{v}$ and $p$, defined in $] 0, T[\times \Omega$, such that

$$
\begin{aligned}
W e \underset{\boldsymbol{\tau}}{\square}+\kappa\left(\left|\boldsymbol{\tau}_{d}\right|\right) \boldsymbol{\tau}-2 \alpha D(\boldsymbol{v}) & =0 \text { in }] 0, T[\times \Omega, \\
R e\left(\frac{\partial \boldsymbol{v}}{\partial t}+(\boldsymbol{v} \cdot \nabla) \boldsymbol{v}\right)-\operatorname{div} \boldsymbol{\tau}-(1-\alpha) \Delta \boldsymbol{v}+\nabla p & =0 \text { in }] 0, T[\times \Omega, \\
-\operatorname{div} \boldsymbol{v} & =0 \text { in }] 0, T[\times \Omega .
\end{aligned}
$$

with some general boundary conditions suitable for practical flows:

$$
\begin{array}{rll}
\boldsymbol{v}=\boldsymbol{v}_{\Gamma} & \text { on } & ] 0, T\left[\times \Gamma_{d},\right. \\
\boldsymbol{\sigma} . \boldsymbol{n}=0 & \text { on } & ] 0, T\left[\times \Gamma_{n},\right. \\
\boldsymbol{v} . \boldsymbol{n}=0 \text { and } \boldsymbol{\sigma}_{n t}=0 & \text { on } & ] 0, T\left[\times \Gamma_{s},\right.
\end{array}
$$

where $\kappa(\xi)=\max (0,1-B i / \xi)$ is now expressed in dimensionless form, $\boldsymbol{v}_{\Gamma}$ is known, $\boldsymbol{n}$ is the unit outward normal on $\partial \Omega$ and $\boldsymbol{\sigma}_{n t}=\boldsymbol{\sigma} \cdot \boldsymbol{n}-\sigma_{n n} . \boldsymbol{n}$ is the tangential stress vector while $\sigma_{n n}=(\boldsymbol{\sigma} . \boldsymbol{n}) . \boldsymbol{n}$ is the normal stress (scalar). The domains $\Gamma_{d}$ and $\Gamma_{n}$ are boundaries with the classical Dirichlet and Neumann type boundary conditions, and $\Gamma_{s}$ is a boundary with slip boundary condition; although rare in the context of fluid mechanics, such a boundary condition is pertinent in the case of foam modeling (see section 4). In addition, since (2) is a transport equation, the extra stress tensor $\tau$ may also be prescribed at the upstream boundary $\Gamma_{-}=\{x \in \partial \Omega, \boldsymbol{v} . \boldsymbol{n}<0\}$ as:

$$
\left.\boldsymbol{\tau}=\boldsymbol{\tau}^{-} \quad \text { on } \quad\right] 0, T\left[\times \Gamma_{-} .\right.
$$

Fig. 1 is an illustration of such boundary conditions.

In the general case, the quantity $p$ is the Lagrangian multiplier associated with the incompressibility constraint (4), it coincides with the physical pressure $p_{\text {tot }}=-1 / 3 \operatorname{tr}(\boldsymbol{\sigma})$ when the elastic stress tensor $\boldsymbol{\tau}$ is traceless, i.e. when $a=0$ in the objective derivative (1).

Notice that when $W e=B i=0$, then $\kappa=1$ and the problem reduces to the usual Navier-Stokes equations with $\boldsymbol{\tau}=2 \alpha D(\boldsymbol{v})$. When $W e>0$ and $B i=0$ the viscoelastic Oldroyd [32] model is obtained. Conversely, when $B i>0$ and $W e=0$, the system reduces to the viscoplastic Bingham $[4,31]$ model. In the case of slow flows, the nonlinear inertia term $(\boldsymbol{v} \cdot \nabla) \boldsymbol{v}$ in (3) can be neglected, which we do in the following.

\section{Numerical method}

The numerical method is based on an adaptation to elastoviscoplastic fluids of a splitting algorithm introduced in $[38,39,40]$ for the viscoelastic fluids. This 
adaptation is not straightforward: while the resulting subproblems at each time steps were linear for viscoelastic fluids, they become here strongly nonlinear.

We describe in details the algorithm below. It is summarized in section 3.4.

\subsection{Time discretization}

Let $\mathcal{U}=(\boldsymbol{\tau}, \boldsymbol{v}, p)$ and $\mathcal{M}=\operatorname{diag}(W e, R e, 0)$. The time-dependent problem (2)-(4) is rewritten in a concise form:

$$
\mathcal{M} \frac{\partial \mathcal{U}}{\partial t}+\mathcal{A}(\mathcal{U})=0
$$

where $\mathcal{A}$ denotes the following nonlinear operator:

$$
\mathcal{A}(\mathcal{U})=\left(\begin{array}{c}
W e\left((\boldsymbol{v} \cdot \nabla) \boldsymbol{\tau}+\beta_{a}(\boldsymbol{\tau}, \nabla \boldsymbol{v})\right)+\kappa\left(\left|\boldsymbol{\tau}_{d}\right|\right) \boldsymbol{\tau}-2 \alpha D(\boldsymbol{v}) \\
-\operatorname{div} \boldsymbol{\tau}-(1-\alpha) \Delta \boldsymbol{v}+\nabla p \\
-\operatorname{div} \boldsymbol{v}
\end{array}\right)
$$

We consider the following operator splitting $\mathcal{A}=\mathcal{A}_{1}+\mathcal{A}_{2}$ where

$$
\mathcal{A}_{1}(\mathcal{U})=\left(\begin{array}{c}
\kappa\left(\left|\boldsymbol{\tau}_{d}\right|\right) \boldsymbol{\tau}-2 \alpha D(\boldsymbol{v}) \\
-\operatorname{div} \boldsymbol{\tau}-(1-\alpha) \Delta \boldsymbol{v}+\nabla p \\
-\operatorname{div} \boldsymbol{v}
\end{array}\right) \quad \text { and } \quad \mathcal{A}_{2}(\mathcal{U})=\left(\begin{array}{c}
W e\left((\boldsymbol{v} \cdot \nabla) \boldsymbol{\tau}+\beta_{a}(\boldsymbol{\tau}, \nabla \boldsymbol{v})\right) \\
0 \\
0
\end{array}\right)
$$

We obtain a splitting of the two main non-linearities: $\mathcal{A}_{1}$ contains all the viscoplastic effects, controlled by the $B i$ number in $\kappa$ and $\mathcal{A}_{2}$ involves the viscoelastic effects, controlled by the $W e$ number. This choice will lead to a decoupled algorithm for the computation of the $(\boldsymbol{v}, p)$ and $\boldsymbol{\tau}$ components. Following [39], let us consider the three-steps $\theta$-scheme time-approximation of the previous equation:

$$
\begin{aligned}
\mathcal{M} \frac{\mathcal{U}^{n+\theta}-\mathcal{U}^{n}}{\theta \Delta t}+\mathcal{A}_{1}\left(\mathcal{U}^{n+\theta}\right)+\mathcal{A}_{2}\left(\mathcal{U}^{n}\right) & =0 \\
\mathcal{M} \frac{\mathcal{U}^{n+1-\theta}-\mathcal{U}^{n+\theta}}{(1-2 \theta) \Delta t}+\mathcal{A}_{1}\left(\mathcal{U}^{n+\theta}\right)+\mathcal{A}_{2}\left(\mathcal{U}^{n+1-\theta}\right) & =0 \\
\mathcal{M} \frac{\mathcal{U}^{n+1}-\mathcal{U}^{n+1-\theta}}{\theta \Delta t}+\mathcal{A}_{1}\left(\mathcal{U}^{n+1}\right)+\mathcal{A}_{2}\left(\mathcal{U}^{n+1-\theta}\right) & =0
\end{aligned}
$$

where $\Delta t>0$ and $\theta \in] 0,1 / 2\left[\right.$ is the scheme parameter. At each time step, $\mathcal{U}^{n}$ is known and we compute successively $\mathcal{U}^{n+\theta}, \mathcal{U}^{n+1-\theta}$ and $\mathcal{U}^{n+1}$ by solving subproblems related to $\mathcal{A}_{1}, \mathcal{A}_{2}$ and $\mathcal{A}_{1}$, respectively, i.e. viscoplastic, viscoelastic and also viscoplastic subproblems.

These subproblems can be rearranged in order to decouple the computation of $(\boldsymbol{v}, p)$ and $\boldsymbol{\tau}$.

Remark that the third step of the $\theta$-scheme is similar to the first one. 


\subsection{The nonlinear Stokes subproblem}

$\boldsymbol{\tau}^{n}$ and $\boldsymbol{v}^{n}$ being known, the first subproblem writes:

$(Q 1)$ : find $\boldsymbol{\tau}^{n+\theta}, \boldsymbol{v}^{n+\theta}$ and $p^{n+\theta}$, defined in $\Omega$, such that

$$
\begin{aligned}
W e \frac{\boldsymbol{\tau}^{n+\theta}}{\theta \Delta t}+\kappa\left(\left|\boldsymbol{\tau}_{d}^{n+\theta}\right|\right) \boldsymbol{\tau}^{n+\theta}-2 \alpha D\left(\boldsymbol{v}^{n+\theta}\right) & =\boldsymbol{\gamma}^{n} \\
\operatorname{Re} \frac{\boldsymbol{v}^{n+\theta}}{\theta \Delta t}-\operatorname{div} \boldsymbol{\tau}^{n+\theta}-(1-\alpha) \Delta \boldsymbol{v}^{n+\theta}+\nabla p^{n+\theta} & =\operatorname{Re} \frac{\boldsymbol{v}^{n}}{\theta \Delta t} \\
-\operatorname{div} \boldsymbol{v}^{n+\theta} & =0
\end{aligned}
$$

completed by the boundary conditions (5)-(7) at step $n+\theta$. where the righthand side $\gamma^{n}$ is explicitly known:

$$
\boldsymbol{\gamma}^{n}=W e\left(\frac{\boldsymbol{\tau}^{n}}{\theta \Delta t}-\left(\boldsymbol{v}^{n} \cdot \nabla\right) \boldsymbol{\tau}^{n}-\beta_{a}\left(\boldsymbol{\tau}^{n}, \nabla \boldsymbol{v}^{n}\right)\right)
$$

Observe that $\kappa=1$ for $B i=0$ and this subproblem reduces to the three-fields formulation of the Stokes problem. When $B i>0$, the $\kappa$ function introduces a strong nonlinearity. Nevertheless, this system can then be solved by a suitable nonlinear algorithm such as a fixed-point method.

Fixed-point algorithm. Let $\left(\overline{\boldsymbol{\tau}}^{k}, \overline{\boldsymbol{v}}^{k}, \bar{p}^{k}\right)_{k \geq 0}$ be the sequence defined by the following induction.

- step $k=0$ : Let $\left(\overline{\boldsymbol{\tau}}^{0}, \overline{\boldsymbol{v}}^{0}, \bar{p}^{0}\right)=\left(\boldsymbol{\tau}^{n}, \boldsymbol{v}^{n}, p^{n}\right)$.

- step $k \geq 1$ : Let $\left(\overline{\boldsymbol{\tau}}^{k-1}, \overline{\boldsymbol{v}}^{k-1}, \bar{p}^{k-1}\right)$ being known. Then we solve the following linear three-field Stokes problem:

$(Q F P)$ : find $\left(\overline{\boldsymbol{\tau}}^{k}, \overline{\boldsymbol{v}}^{k}, \bar{p}^{k}\right)$ such that

$$
\begin{aligned}
W e \frac{\overline{\boldsymbol{\tau}}^{k}}{\theta \Delta t}+\kappa\left(\left|\overline{\boldsymbol{\tau}}_{d}^{k-1}\right|\right) \overline{\boldsymbol{\tau}}^{k}-2 \alpha D\left(\overline{\boldsymbol{v}}^{k}\right) & =\gamma^{n} \\
\operatorname{div} \overline{\boldsymbol{\tau}}^{k}-\frac{R e}{\theta \Delta t} \overline{\boldsymbol{v}}^{k}+(1-\alpha) \Delta \overline{\boldsymbol{v}}^{k}-\nabla \bar{p}^{k} & =-\operatorname{Re} \frac{\boldsymbol{v}^{n}}{\theta \Delta t} \\
\operatorname{div} \overline{\boldsymbol{v}}^{k} & =0
\end{aligned}
$$

completed by the boundary conditions at step $k$, replacing in (5)-(7) $\boldsymbol{v}$ by $\overline{\boldsymbol{v}}^{k}$ and $\boldsymbol{\sigma}$ by $-\bar{p}^{k} I+2(1-\alpha) D\left(\overline{\boldsymbol{v}}^{k}\right)+\overline{\boldsymbol{\tau}}^{k}$.

- Arrest when a criterium on the residual of the initial nonlinear problem $Q 1$ is satisfied. This criterium is defined at the discrete level (see eq.(21) below).

The linear subproblem in each step of the fixed point algorithm is a threefields Stokes problem. The sequence $\left(\overline{\boldsymbol{\tau}}^{k}, \overline{\boldsymbol{v}}^{k}, \bar{p}^{k}\right)_{k \geq 0}$ of the fixed-point algorithm converges to $\left(\boldsymbol{\tau}^{n+\theta}, \boldsymbol{v}^{n+\theta}, p^{n+\theta}\right)$, the solution of the first subproblem (9)-(11). We describe now the variational formulation of this subproblem. 
Variational formulation of the linearized three-fields Stokes subproblem. We introduce

$$
\begin{gathered}
T=\left\{\boldsymbol{\tau}=\left(\tau_{i j}\right) ; \tau_{i j}=\tau_{j i} ; \tau_{i j} \in L^{2}(\Omega) ; i, j=1, \ldots, N\right\}, \\
X(\mathbf{u})=\left\{\boldsymbol{v} \in H^{1}(\Omega)^{N}, \boldsymbol{v}=\mathbf{u} \operatorname{sur} \Gamma_{c}, \boldsymbol{v} \cdot \boldsymbol{n}=0 \text { on } \Gamma_{s}\right\}, \\
Q=L_{0}^{2}(\Omega)=\left\{q \in L^{2}(\Omega) ; \int_{\Omega} q=0\right\}
\end{gathered}
$$

and we denote by

$$
(p, q)=\int_{\Omega} p q(x) \mathrm{d} x, \quad(\boldsymbol{v}, \boldsymbol{w})=\int_{\Omega} \boldsymbol{v} \cdot \boldsymbol{w}(x) \mathrm{d} x, \quad(\boldsymbol{\tau}, \boldsymbol{\gamma})=\int_{\Omega} \boldsymbol{\tau}: \boldsymbol{\gamma}(x) \mathrm{d} x
$$

the scalar products on $L^{2}(\Omega), L^{2}(\Omega)^{N}, L^{2}(\Omega)^{N^{2}}$, and $\|\cdot\|$ the corresponding norms. In the following, $C_{1}, C_{2}, C_{3}, C_{4}, C_{5}$ are strictly positive real constants. Denoting $\nu\left(\overline{\boldsymbol{\tau}}^{k-1}\right)=\left(\frac{W e}{\theta \Delta t}+\kappa\left(\left|\overline{\boldsymbol{\tau}}_{d}^{k-1}\right|\right)\right)$, a variational formulation of problem $(Q F P)$ writes

$(V F Q F P)$ : find $\left(\overline{\boldsymbol{\tau}}^{k}, \overline{\boldsymbol{v}}^{k}, \bar{p}^{k}\right) \in T \times X\left(\boldsymbol{v}_{\Gamma}\right) \times Q$ such that

$$
\begin{aligned}
\left(\nu\left(\overline{\boldsymbol{\tau}}_{k-1}\right) \overline{\boldsymbol{\tau}}^{k}, \boldsymbol{\gamma}\right)-2 \alpha\left(D\left(\overline{\boldsymbol{v}}^{k}\right), \boldsymbol{\gamma}\right) & =\left(\boldsymbol{\gamma}^{n}, \boldsymbol{\gamma}\right), \\
\left(\frac{R e}{\theta \Delta t}\right)\left(\overline{\boldsymbol{v}}^{k}, \boldsymbol{w}\right)+2(1-\alpha)\left(D\left(\overline{\boldsymbol{v}}^{k}\right), D(\boldsymbol{w})\right) & \\
+\left(\overline{\boldsymbol{\tau}}^{k}, D(\boldsymbol{w})\right)-\left(\bar{p}^{k}, \operatorname{div} \mathbf{w}\right) & =\frac{\operatorname{Re}}{\theta \Delta t}\left(\boldsymbol{v}^{n}, \boldsymbol{w}\right), \\
\left(q, \operatorname{div} \overline{\boldsymbol{v}}^{k}\right) & =0,
\end{aligned}
$$

$\forall(\boldsymbol{\gamma}, \boldsymbol{w}, q) \in T \times X(\mathbf{0}) \times Q$. Following Baranger and Sandri [2], we write this problem in the form

Find $\left(\overline{\boldsymbol{\tau}}^{k}, \overline{\boldsymbol{v}}^{k}, \bar{p}^{k}\right) \in T \times X\left(\boldsymbol{v}_{\Gamma}\right) \times Q$ such that

$$
\begin{aligned}
a\left(\left(\overline{\boldsymbol{\tau}}^{k}, \overline{\boldsymbol{v}}^{k}\right),(\boldsymbol{\gamma}, \boldsymbol{w})\right)+b\left(\boldsymbol{w}, \bar{p}^{k}\right) & =\left(\boldsymbol{\gamma}^{n}, \boldsymbol{\gamma}\right)+2 \alpha \frac{R e}{\theta \Delta t}\left(\boldsymbol{v}^{n}, \boldsymbol{w}\right), \\
b\left(\overline{\boldsymbol{v}}^{k}, q\right) & =0, \\
\forall(\boldsymbol{\gamma}, \boldsymbol{w}, q) \in T & \times X(\mathbf{0}) \times Q,
\end{aligned}
$$

where

$$
\begin{aligned}
a\left(\left(\overline{\boldsymbol{\tau}}^{k}, \overline{\boldsymbol{v}}^{k}\right),(\boldsymbol{\gamma}, \boldsymbol{w})\right)= & \left(\nu\left(\overline{\boldsymbol{\tau}}^{k-1}\right) \overline{\boldsymbol{\tau}}^{k}, \boldsymbol{\gamma}\right)-2 \alpha\left(D\left(\overline{\boldsymbol{v}}^{k}\right), \boldsymbol{\gamma}\right)+2 \alpha\left(\frac{R e}{\theta \Delta t}\right)\left(\overline{\boldsymbol{v}}^{k}, \boldsymbol{w}\right) \\
& +4 \alpha(1-\alpha)\left(D\left(\overline{\boldsymbol{v}}^{k}\right), D(\boldsymbol{w})\right)+2 \alpha\left(\overline{\boldsymbol{\tau}}^{k}, D(\boldsymbol{w})\right)
\end{aligned}
$$

and $b\left(\boldsymbol{w}, \bar{p}^{k}\right)=-\left(\bar{p}^{k}, \operatorname{div} \boldsymbol{w}\right)$. 
One can easily show that the bilinear form $a$ is coercive and continuous on $X(\mathbf{0})$ for $0<\alpha<1$; as in addition, there is an inf-sup condition between the space of velocity with a slip boundary condition $X(\mathbf{0})$ and the pressure space $Q$ [47], problem $(V F Q F P)$ admits a unique solution for $0<\alpha<1$.

If $\alpha=1$, one can still prove the unicity of the solution, and its existence in discrete spaces.

Space discretization. Following [14], the space discretization of the three field Stokes system (12)-(14) is based on a discontinuous piecewise affine finite element method for stresses, while velocities and pressures are approximated by the Taylor-Hood $P_{2}-P_{1}$ element. Since stresses approximation is discontinuous, the transport subproblem will be discretized by the discontinuous Galerkin method, suitable for first-order hyperbolic systems [26, 2] (see section 3.3).

Let $\Omega_{h}$ be a polygonal approximation of the domain $\Omega \mathcal{T}_{h}$ a triangulation of $\Omega_{h}$. The boundary $\Gamma_{h}$ of $\Omega_{h}$ is partitioned into three subdomains $\Gamma_{d, h}, \Gamma_{n, h}, \Gamma_{s, h}$ that correspond to $\Gamma_{d}, \Gamma_{n}, \Gamma_{s}$. Let $T_{h}, X_{h}(\mathbf{u})$, and $Q_{h}$ be the finite element spaces corresponding to $T, X(\mathbf{u})$ et $Q$.

We introduce

$$
\widetilde{X}_{h}=\left\{\boldsymbol{v}_{h}: \Omega_{h} \rightarrow \mathbb{R}^{2} \mid \boldsymbol{v}_{h} \in C\left(\bar{\Omega}_{h}\right)^{2}, \boldsymbol{v}_{h} \in \mathcal{P}_{2}(K)^{2}, \forall K \in \mathcal{T}_{h}\right\} .
$$

and we choose

$$
Q_{h}=\left\{q_{h}: \Omega_{h} \rightarrow \mathbb{R} \mid q_{h} \in C\left(\bar{\Omega}_{h}\right), q_{h} \in \mathcal{P}_{1}(K), \forall K \in \mathcal{T}_{h}\right\} .
$$

This combination of spaces verifies the Babuska-Brezzi conditions [16], but we have to take into account the slip boundary condition. We denote by $\mathcal{N}_{h}$ the set of vertices of $\mathcal{T}_{h}$ and of the middles of the edges of the triangles in $\mathcal{T}_{h}$, and $\boldsymbol{n}_{h}$ an approximation of the unit normal $\boldsymbol{n}$. We choose

$$
X_{h}\left(\mathbf{u}_{h}\right)=\left\{\boldsymbol{v}_{h} \in \widetilde{X}_{h}\left|\boldsymbol{v}_{h}\right| \Gamma_{d, h}=\mathbf{u}_{h}, \boldsymbol{v}_{h}\left(x_{s}\right) \cdot \boldsymbol{n}_{h}\left(x_{s}\right)=0, \forall x_{s} \in \mathcal{N}_{h} \cap \Gamma_{s, h}\right\} .
$$

If $\boldsymbol{n}_{h}$ is defined for the projection $P_{\Gamma}$ from $\Gamma_{s}$ to $\Gamma_{s, h}$ :

$$
\boldsymbol{n}_{h}\left(x_{s}\right)=\boldsymbol{n}\left(P_{\Gamma}\left(x_{s}\right)\right), \quad \forall x_{s} \in \mathcal{N}_{h} \cap \Gamma_{s, h},
$$

one can show $[1,46]$ that the spaces $X_{h}(\mathbf{0})$ and $Q_{h}$ verify the Babuska-Brezzi conditions.

The space for stresses is defined as

$$
T_{h}=\left\{\boldsymbol{\tau} \in T ; \boldsymbol{\tau}_{\mid K} \in \mathcal{P}_{1}(K)^{4}, \forall K \in \mathcal{T}_{h}\right\} .
$$

Matrix formulation of $(V F Q F P)$. We introduce the following matrices

- the matrices $M_{t}$ and $M\left(\nu_{h}\left(\overline{\boldsymbol{\tau}}_{h}^{k}\right)\right)$ are such that, $\forall \overline{\boldsymbol{\tau}}_{h}^{k}, \gamma_{h} \in T_{h}$,

$$
\boldsymbol{\gamma}_{h}^{T} M_{t} \overline{\boldsymbol{\tau}}_{h}^{k}=\left(\overline{\boldsymbol{\tau}}_{h}^{k}, \boldsymbol{\gamma}_{h}\right) ; \quad \boldsymbol{\gamma}_{h}^{T} M\left(\nu_{h}\left(\overline{\boldsymbol{\tau}}_{h}^{k-1}\right)\right) \overline{\boldsymbol{\tau}}_{h}^{k}=\left(\nu_{h}\left(\overline{\boldsymbol{\tau}}_{h}^{k-1}\right) \overline{\boldsymbol{\tau}}_{h}^{k}, \boldsymbol{\gamma}_{h}\right) ;
$$


- the matrices $M_{v}$ et $A$ are such that, $\forall \overline{\boldsymbol{v}}_{h}^{k} \in X_{h}\left(\overline{\boldsymbol{v}}_{d, h}\right)$ and $\forall \boldsymbol{w}_{h} \in X_{h}\left(\boldsymbol{w}_{d, h}\right)$,

$$
\boldsymbol{w}_{h}^{T} M_{v} \overline{\boldsymbol{v}}_{h}^{k}=\left(\boldsymbol{w}_{h}, \overline{\boldsymbol{v}}_{h}^{k}\right) ; \quad \boldsymbol{w}_{h}^{T} A \overline{\boldsymbol{v}}_{h}^{k}=2\left(D\left(\overline{\boldsymbol{v}}_{h}^{k}\right), D\left(\boldsymbol{w}_{h}\right)\right) ;
$$

- the matrix $B$ is such that, $\forall \overline{\boldsymbol{v}}_{h}^{k} \in X_{h}\left(\overline{\boldsymbol{v}}_{d, h}\right)$ and $\forall \gamma_{h} \in T_{h}$,

$$
\boldsymbol{\gamma}_{h}^{T} B \overline{\boldsymbol{v}}_{h}^{k}=-2\left(D\left(\overline{\boldsymbol{v}}_{h}^{k}\right), \boldsymbol{\gamma}_{h}\right)
$$

- the matrix $C$ is such that, $\forall \overline{\boldsymbol{v}}_{h}^{k} \in X_{h}\left(\overline{\boldsymbol{v}}_{d, h}\right)$ and $\forall \bar{p}_{h}^{k} \in Q_{h}$,

$$
\left(\bar{p}_{h}^{k}\right)^{T} C \overline{\boldsymbol{v}}_{h}^{k}=-\left(\bar{p}_{h}^{k}, \operatorname{div} \overline{\boldsymbol{v}}_{h}^{k}\right)
$$

- the matrix $M_{p}$ is such that $\forall \bar{p}_{h}^{k}, q_{h} \in Q_{h}$,

$$
q_{h}^{T} M_{p} q_{h}=\left(\bar{p}_{h}^{k}, q_{h}\right) .
$$

The problem $(V F Q F P)$ writes

Find $\left(\overline{\boldsymbol{\tau}}_{h}^{k}, \overline{\boldsymbol{v}}_{h}^{k}, \bar{p}_{h}^{k}\right)$ such that

$$
\begin{aligned}
M\left(\nu_{h}\left(\overline{\boldsymbol{\tau}}_{h}^{k-1}\right)\right) \overline{\boldsymbol{\tau}}_{h}^{k}+\alpha B \overline{\boldsymbol{v}}_{h}^{k} & =M_{t} T_{h}^{n}, \\
\left(\frac{R e}{\theta \Delta t}+C_{F}\right) M_{v} \overline{\boldsymbol{v}}_{h}^{k}+(1-\alpha) A \overline{\boldsymbol{v}}_{h}^{k} & \\
-1 / 2 B^{T} \overline{\boldsymbol{\tau}}_{h}^{k}+C \bar{p}_{h}^{k} & =\frac{R e}{\theta \Delta t} M_{v} \overline{\boldsymbol{v}}_{h}^{n}, \\
C^{T} \overline{\boldsymbol{v}}_{h}^{k}=0 . &
\end{aligned}
$$

The matrix $M\left(\nu_{h}\left(\overline{\boldsymbol{\tau}}_{h}^{k-1}\right)\right)$ is built on the space $T_{h}$ of stresses, which components are $\mathcal{P}_{1}$ discontinuous function on each simplex. As a consequence, this matrix is block-diagonal, with for instance $3 \times 3$ blocks in the case of triangular elements, and can be inverted at a low cost. Therefore, one can express directly $\overline{\boldsymbol{\tau}}_{h}^{k}$ from $D\left(\overline{\boldsymbol{v}}_{h}^{k}\right)$ :

$$
\overline{\boldsymbol{\tau}}_{h}^{k}=M\left(\nu_{h}\left(\overline{\boldsymbol{\tau}}_{h}^{k-1}\right)\right)^{-1}\left(M_{t} T^{n}-\alpha B \overline{\boldsymbol{v}}_{h}^{k}\right) .
$$

And we have

$$
\left(\begin{array}{cc}
M & C \\
C^{T} & 0
\end{array}\right)\left(\begin{array}{c}
\overline{\boldsymbol{v}}_{h}^{k} \\
\bar{p}_{h}^{k}
\end{array}\right)=F
$$

where

$$
M=\left(\frac{R e}{\theta \Delta t}+C_{F}\right) M_{v}+(1-\alpha) A+\frac{\alpha}{2} B^{T} M\left(\nu_{h}\left(\overline{\boldsymbol{\tau}}_{h}^{k-1}\right)\right)^{-1} B
$$

and

$$
F=\left(\begin{array}{c}
\frac{R e}{\theta \Delta t} M_{v} \overline{\boldsymbol{v}}^{n}+1 / 2 B^{T} M\left(\nu_{h}\left(\overline{\boldsymbol{\tau}}_{h}^{k-1}\right)\right)^{-1} M_{t} T^{n} \\
0
\end{array}\right)
$$


Stopping criterium for the fixed-point algorithm. The fixed-point algorithm is expected to converge to a discrete solution that satisfies the following nonlinear discrete problem:

$$
\begin{aligned}
& \text { find }\left(\boldsymbol{\tau}_{h}, \boldsymbol{v}_{h}, p_{h}\right) \in T_{h} \times X_{h}\left(\mathbf{u}_{h}^{D}\right) \times Q_{h} \text { such that } \\
& \qquad \begin{aligned}
M\left(\nu_{h}\left(\boldsymbol{\tau}_{h}\right)\right) \boldsymbol{\tau}_{h}+\alpha B \boldsymbol{v}_{h} & =M_{t} T_{h}^{n}, \\
\left(\frac{R e}{\theta \Delta t}+C_{F}\right) M_{v} \boldsymbol{v}_{h}+(1-\alpha) A \boldsymbol{v}_{h} & \\
-1 / 2 B^{T} \boldsymbol{\tau}_{h}+C p_{h} & =\frac{R e}{\theta \Delta t} M_{v} \boldsymbol{v}^{n}, \\
C^{T} \boldsymbol{v}_{h}=0 . &
\end{aligned}
\end{aligned}
$$

Therefore, the residual terms of the $k$-th iteration of the fixed-point algorithm are defined by:

$$
\begin{aligned}
\rho_{F P, \boldsymbol{\tau}}^{k} & =M_{t}^{-1}\left[M\left(\nu_{h}\left(\overline{\boldsymbol{\tau}}_{h}^{k}\right)\right) \overline{\boldsymbol{\tau}}_{k, h}+\alpha B \overline{\boldsymbol{v}}_{k, h}-M_{t} \bar{T}_{h}^{n}\right], \\
\rho_{F P, \boldsymbol{v}}^{k} & =M_{v}^{-1}\left[\left(\frac{R e}{\theta \Delta t}+C_{F}\right) M_{v}\left(\overline{\boldsymbol{v}}_{k, h}-\overline{\boldsymbol{v}}_{h}^{n}\right)+(1-\alpha) A \overline{\boldsymbol{v}}_{k, h}-1 / 2 B^{T} \overline{\boldsymbol{\tau}}_{k, h}+C \bar{p}_{k, h}\right], \\
\rho_{F P, p}^{k} & =M_{p}^{-1}\left[C^{T} \overline{\boldsymbol{v}}_{k, h}\right] .
\end{aligned}
$$

Let $\varepsilon_{F P}$ be a strictly positive real number, the stopping criterium is then given by:

$$
\left\|\rho_{F P, \boldsymbol{\tau}}^{k}\right\|+\left\|\rho_{F P, \boldsymbol{v}}^{k}\right\|+\left\|\rho_{F P, p}^{k}\right\| \leq \varepsilon_{F P} .
$$

\subsection{The stress transport subproblem}

This subproblem is more classic in the context of viscoelastic fluid flow problems. Assume $W e>0$ and let $\theta^{\prime}=1-2 \theta$. Expanding $\mathcal{A}_{1}$ and $\mathcal{A}_{2}$, the second step of the $\theta$-scheme writes:

$(Q 2)$ : find $\boldsymbol{\tau}^{n+1-\theta}$ and $\boldsymbol{v}^{n+1-\theta}$, defined in $\Omega$, such that

$$
\begin{aligned}
& \frac{\boldsymbol{\tau}^{n+1-\theta}}{\theta^{\prime} \Delta t}+\left(\boldsymbol{v}^{n+1-\theta} \cdot \nabla\right) \boldsymbol{\tau}^{n+1-\theta}+\beta_{a}\left(\boldsymbol{\tau}^{n+1-\theta}, \nabla \boldsymbol{v}^{n+1-\theta}\right)=\delta^{n+\theta} \\
& \frac{R e}{\theta^{\prime} \Delta t} \boldsymbol{v}^{n+1-\theta}=\frac{R e}{\theta^{\prime} \Delta t} \boldsymbol{v}^{n+\theta}+\operatorname{div} \boldsymbol{\tau}^{n+\theta}+(1-\alpha) \Delta \boldsymbol{v}^{n+\theta}-\nabla p^{n+\theta}
\end{aligned}
$$

together with the upstream boundary condition (8) for $\boldsymbol{\tau}^{n+1-\theta}$, and where

$$
\delta^{n+\theta}=\frac{1}{\theta^{\prime} \Delta t} \boldsymbol{\tau}^{n+\theta}-\frac{\kappa\left(\left|\boldsymbol{\tau}_{d}^{n+\theta}\right|\right)}{W e} \boldsymbol{\tau}^{n+\theta}+\frac{2 \alpha}{W e} D\left(\boldsymbol{v}^{n+\theta}\right)
$$

Well-posedness of the transport problem. Subtracting (10) to (23) and assuming $R e>0$ leads to an explicit expression for $\boldsymbol{v}^{n+1-\theta}$ :

$$
\boldsymbol{v}^{n+1-\theta}=\frac{1-\theta}{\theta} \boldsymbol{v}^{n+\theta}-\frac{1-2 \theta}{\theta} \boldsymbol{v}^{n}
$$


As this expression does not involve $R e$, we use it even in the case $R e=0$. Since $\boldsymbol{v}^{n+1-\theta}$ is known, (22) is a linear Friedrich's first-order system [15] for $\boldsymbol{\tau}^{n+1-\theta}$. Let $L^{2}(\Omega)_{s}^{d \times d}$ be the space of symmetric tensors defined in $\Omega$ and $T$ the linear operator defined for all $\tau \in L^{2}(\Omega)_{s}^{d \times d}$ by

$$
T(\boldsymbol{\tau})=\frac{\boldsymbol{\tau}}{\theta^{\prime} \Delta t}+\left(\boldsymbol{v}^{n+1-\theta} \cdot \nabla\right) \boldsymbol{\tau}+\beta_{a}\left(\boldsymbol{\tau}, \nabla \boldsymbol{v}^{n+1-\theta}\right)
$$

The formal adjoint of $T$, denoted as $T^{*}$, is defined for all $\tau \in L^{2}(\Omega)_{s}^{d \times d}$ by

$$
T^{*}(\boldsymbol{\tau})=\frac{\boldsymbol{\tau}}{\theta^{\prime} \Delta t}-\left(\boldsymbol{v}^{n+1-\theta} \cdot \nabla\right) \boldsymbol{\tau}-\beta_{-a}\left(\boldsymbol{\tau}, \nabla \boldsymbol{v}^{n+1-\theta}\right)
$$

Remark that $T^{*}$ is associated to a bilinear form with $-a$ parameter. Let us introduce $T_{s}=\left(T+T^{*}\right) / 2$, the symmetric part of $T$ :

$$
\begin{aligned}
T_{s}(\boldsymbol{\tau}) & =\frac{\boldsymbol{\tau}}{\theta^{\prime} \Delta t}+\frac{1}{2}\left(\beta_{a}-\beta_{-a}\right)\left(\boldsymbol{\tau}, \nabla \boldsymbol{v}^{n+1-\theta}\right) \\
& =\frac{\boldsymbol{\tau}}{\theta^{\prime} \Delta t}-a\left(D\left(\boldsymbol{v}^{n+1-\theta}\right) \boldsymbol{\tau}+\boldsymbol{\tau} D\left(\boldsymbol{v}^{n+1-\theta}\right)\right)
\end{aligned}
$$

For all $\boldsymbol{\tau}, \boldsymbol{\gamma} \in \mathbb{R}_{s}^{d \times d}$, let us denote $\boldsymbol{\tau}: \boldsymbol{\gamma}$ the scalar product in $\mathbb{R}_{s}^{d \times d}$, defined by $\boldsymbol{\tau}: \boldsymbol{\gamma}=\sum_{i, j=1}^{d} \tau_{i, j} \gamma_{i, j}$. Remark that $\boldsymbol{\tau}: \boldsymbol{\tau}=|\boldsymbol{\tau}|^{2}$. From Friedrichs [15], the operator $T$ is said to be positive if there exists a constant $C>0$ such that, for all $\boldsymbol{\tau} \in \mathbb{R}_{s}^{d \times d}$ we have:

$$
T_{s}(\boldsymbol{\tau}): \boldsymbol{\tau} \geq C|\boldsymbol{\tau}|^{2}, \forall \boldsymbol{\tau} \in \mathbb{R}_{s}^{d \times d}
$$

Under this condition, the problem (22), together with the upstream boundary condition (8) for $\boldsymbol{\tau}^{n+1-\theta}$, admits a weak solution [15].

A short computations shows that the positivity writes also:

$$
\frac{1}{\theta^{\prime} \Delta t}-2|a|\left\|D\left(\boldsymbol{v}^{n+1-\theta}\right)\right\|_{\infty}>0
$$

Assuming $\boldsymbol{v}^{n+1-\theta} \in W^{1, \infty}$, remark that when $a=0$ this condition is always satisfied. Otherwise, when $a \neq 0$, this condition expresses that the time step $\Delta t$ may be chosen small enough.

Variational formulation of the stress transport subproblem. As the stress tensor $\boldsymbol{\tau}$ is approximated in a $\mathcal{P}_{1} d$ finite element space, it is natural to consider the Discontinuous Galerkin method for this transport subproblem: for a given simplex $K \in \mathcal{T}_{h}$, let $\boldsymbol{n}_{h}$ be the external normal on its edges. We denote by $\Gamma_{K}^{-}$the part of its boundary where $\boldsymbol{v}_{h}^{n+1-\vartheta} \cdot \boldsymbol{n}_{h}<0$. The solution $\boldsymbol{\tau}^{n+1-\theta}$ of the continuous transport problem $(Q 2)$ is approximated by $\tau_{h}^{n+1-\theta} \in T_{h}$, solution of the discrete problem 
$(V F Q 2)_{h}$ Find $\boldsymbol{\tau}_{h} \in T_{h}$ such that, $\forall \gamma_{h} \in T_{h}$,

$$
\begin{gathered}
\left(\frac{1}{\theta^{\prime} \Delta t} \boldsymbol{\tau}_{h}+\left(\boldsymbol{v}_{h}^{n+1-\theta} \cdot \nabla\right) \boldsymbol{\tau}_{h}+\beta_{a}\left(\boldsymbol{\tau}_{h}, \nabla \boldsymbol{v}_{h}^{n+1-\theta}\right), \gamma_{h}\right) \\
-\sum_{K \in \mathcal{T}_{h}} \int_{\Gamma_{K}^{-}}\left(\boldsymbol{v}_{h}^{n+1-\theta} \cdot \boldsymbol{n}_{h}\right)\left(\left(\boldsymbol{\tau}_{h}-\boldsymbol{\tau}_{e x t}\right): \boldsymbol{\gamma}_{h}\right) \mathrm{d} s=\left(\delta_{h}^{n+\theta}, \boldsymbol{\gamma}_{h}\right) \\
-\int_{\Gamma_{h}^{-}}\left(\boldsymbol{v}_{h}^{n+1-\theta} \cdot \boldsymbol{n}_{h}\right)\left(\boldsymbol{\tau}_{h}^{-}: \boldsymbol{\gamma}_{h}\right) \mathrm{d} s,
\end{gathered}
$$

where

$$
\boldsymbol{\tau}_{\text {ext }}=\left\{\begin{array}{c}
0 \text { if } \Gamma_{K}^{-} \subset \Gamma_{h}, \\
\text { external trace of } \boldsymbol{\tau}_{h} \text { on } \Gamma_{K}^{-} \text {otherwise. }
\end{array}\right.
$$

The second term of the right-hand side enforces the inbound flux condition (8) on $\Gamma_{h}^{-}$.

Matrix formulation. For $\mathbf{u}_{h} \in X_{h}\left(\boldsymbol{v}_{h}^{D}\right)$, let $G\left(\mathbf{u}_{h}\right)$ be the matrix such that $\forall \tau_{h}, \gamma_{h} \in T_{h}$,

$$
\boldsymbol{\gamma}_{h}^{T} G\left(\mathbf{u}_{h}\right) \boldsymbol{\tau}_{h}=\left(\left(\mathbf{u}_{h} \cdot \nabla\right) \boldsymbol{\tau}_{h}, \boldsymbol{\gamma}_{h}\right)-\sum_{K \in \mathcal{T}_{h}} \int_{\Gamma_{K}^{-}}\left(\mathbf{u}_{h} \cdot \boldsymbol{n}_{h}\right)\left(\left(\boldsymbol{\tau}_{h}-\boldsymbol{\tau}_{e x t}\right): \boldsymbol{\gamma}_{h}\right) \mathrm{d} s
$$

We denote by $B_{a}\left(\mathbf{u}_{h}\right)$ the matrix such that $\forall \boldsymbol{\tau}_{h}, \gamma_{h} \in T_{h}$,

$$
\boldsymbol{\gamma}_{h}^{T} B_{a}\left(\mathbf{u}_{h}\right) \boldsymbol{\tau}_{h}=\left(\beta_{a}\left(\boldsymbol{\tau}_{h}, \nabla \boldsymbol{v}_{h}^{n+1-\theta}\right), \boldsymbol{\gamma}_{h}\right) .
$$

Finally, let $F^{-} \in Y_{h}$ be defined as

$$
F^{-}=-W e \int_{\Gamma_{h}^{-}}\left(\boldsymbol{v}_{h}^{n+1-\theta} \cdot \boldsymbol{n}_{h}\right)\left(\boldsymbol{\tau}_{h}^{-}: \gamma_{h}\right) \mathrm{d} s
$$

The matrix formulation of the discrete transport problem $(V F Q 2)_{h}$ writes:

$$
W e G\left(\boldsymbol{v}_{h}^{n+1-\theta}\right) \boldsymbol{\tau}_{h}+W e B_{a}\left(\boldsymbol{v}_{h}^{n+1-\theta}\right) \boldsymbol{\tau}_{h}=M_{t} S_{h}^{n}+F^{-} .
$$

\subsection{The discrete $\theta$-scheme}

We summarize here the discrete formulation of the $\theta$-scheme:

- Initialization : let $\boldsymbol{\tau}_{h}^{0}$ and $\boldsymbol{v}_{h}^{0}$ being known.

- Let $n>0$. Suppose $\boldsymbol{\tau}_{h}^{n}$ and $\boldsymbol{v}_{h}^{n}$ are known. The computation of $\boldsymbol{\tau}_{h}^{n+1}, \boldsymbol{v}_{h}^{n+1}$ and $p_{h}^{n+1}$ involves three steps: first $\left(\boldsymbol{\tau}_{h}^{n+\theta}, \boldsymbol{v}_{h}^{n+\theta}, p_{h}^{n+\theta}\right)$, then $\left(\boldsymbol{\tau}_{h}^{n+1-\theta}, \boldsymbol{v}_{h}^{n+1-\theta}\right)$ and finally $\left(\boldsymbol{\tau}_{h}^{n+1}, \boldsymbol{v}_{h}^{n+1}, p_{h}^{n+1}\right)$ 
- step 1 (fixed point): let us introduce the sequence $\left(\boldsymbol{\tau}_{k, h}, \boldsymbol{v}_{k, h}, p_{k, h}\right)_{k \geq 0}$ defined by:

$k=0:$ set $\boldsymbol{\tau}_{0, h}=\boldsymbol{\tau}_{h}^{n}$ and $\boldsymbol{v}_{0, h}=\boldsymbol{v}_{h}^{n}$

$k \geq 0$ : let $\left(\boldsymbol{\tau}_{k+1, h}, \boldsymbol{v}_{k+1, h}, p_{k+1, h}\right)$ be the solution of the discrete Stokes-like subproblem $(V F Q F P)$. The stopping criterium is:

$$
\left\|\rho_{F P, \boldsymbol{\tau}}^{k}\right\|+\left\|\rho_{F P, \boldsymbol{v}}^{k}\right\|+\left\|\rho_{F P, p}^{k}\right\| \leq \varepsilon_{F P},
$$

and then

$$
\boldsymbol{\tau}_{h}^{n+\theta}=\boldsymbol{\tau}_{k+1, h} ; \quad \boldsymbol{v}_{h}^{n+\theta}=\boldsymbol{v}_{k+1, h} ; \quad p_{h}^{n+\theta}=p_{k+1, h} .
$$

- step 2 (transport): $\boldsymbol{v}_{h}^{n+1-\theta}$ is computed explicitly by:

$$
\boldsymbol{v}^{n+1-\theta}=\frac{1-\theta}{\theta} \boldsymbol{v}^{n+\theta}-\frac{1-2 \theta}{\theta} \boldsymbol{v}^{n}
$$

and $\boldsymbol{\tau}_{h}^{n+1-\theta}$ is characterized as the solution of the transport subproblem (VFQ2)

- step 3: this step is identical to step 1 , by replacing $n+\theta$ by $n+1$ and $n$ by $n+1-\theta$.

- The loop is ended either at a given time or when the stationary solution is reached, which require an appropriate criterium, described below in (28).

Criterium for a stationnary solution. In agreement with the discrete $\theta$ scheme described above, a discrete stationary solution $\left(\boldsymbol{\tau}_{h}, \boldsymbol{v}_{h}, p_{h}\right)$ should verify

$$
\begin{aligned}
\left(W e\left(G\left(\boldsymbol{v}_{h}\right)+B_{a}\left(\boldsymbol{v}_{h}\right)\right)+M\left(\nu_{h}\left(\boldsymbol{\tau}_{h}\right)\right)\right) \boldsymbol{\tau}_{h}-F^{-}+\alpha B \boldsymbol{v}_{h} & =0 \\
\left(C_{F} M_{v}+(1-\alpha) A\right) \boldsymbol{v}_{h}-1 / 2 B^{T} \boldsymbol{\tau}_{h}+C p_{h} & =0 \\
C^{T} \boldsymbol{v}_{h} & =0
\end{aligned}
$$

For a given iteration $\left(\boldsymbol{\tau}_{h}^{n}, \boldsymbol{v}_{h}^{n}, p_{h}^{n}\right)$ of the $\theta$-scheme, we define the residual terms relative to the constitutive equation $\rho_{G, \boldsymbol{\tau}}^{n}$, to the momentum equation $\rho_{G, \boldsymbol{v}}^{n}$, and to the mass conservation $\rho_{G, p}^{n}$ :

$$
\begin{aligned}
\rho_{G, \boldsymbol{\tau}}^{n} & =M_{t}^{-1}\left[\left(W e\left(G\left(\boldsymbol{v}_{h}^{n}\right)+B_{a}\left(\boldsymbol{v}_{h}^{n}\right)\right)+M\left(\nu_{h}\left(\boldsymbol{\tau}_{h}^{n}\right)\right)\right) \boldsymbol{\tau}_{h}^{n}-F^{-}+\alpha B \boldsymbol{v}_{h}^{n}\right](25) \\
\rho_{G, \boldsymbol{v}}^{n} & =M_{v}^{-1}\left[\left(C_{F} M_{v}+(1-\alpha) A\right) \boldsymbol{v}_{h}^{n}-1 / 2 B^{T} \boldsymbol{\tau}_{h}^{n}+C p_{h}^{n}\right], \\
\rho_{G, p}^{n} & =M_{p}^{-1}\left[C^{T} \boldsymbol{v}_{h}^{n}\right] .
\end{aligned}
$$

Let $\varepsilon_{G}$ be a strictly positive real number; the stopping criterium is then given by:

$$
\left\|\rho_{G, \boldsymbol{\tau}}^{n}\right\|+\left\|\rho_{G, \boldsymbol{v}}^{n}\right\|+\left\|\rho_{G, p}^{n}\right\| \leq \varepsilon_{G} .
$$




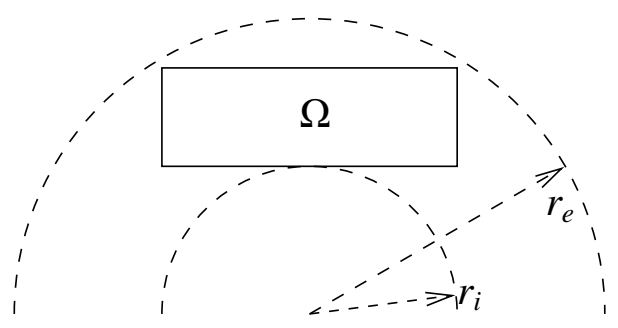

Figure 2: Geometry of the Couette flow and the computational domain.

\section{Results and discussion}

\subsection{Tests, validation and convergence results}

Our $\theta$-scheme algorithm is first checked for a viscoelastic Oldroyd model, obtained with $B i=0$. We consider the exact stationary solution of the Oldroyd-B model, i.e. with the upper convected objective derivative associated to $a=1$, in the Couette flow between two cylinders (see fig. 2). Let $r_{i}$ (resp. $r_{e}$ ) be the radius of the inner (resp. outer) cylinder, and $v_{i}$ (resp. $v_{e}$ ) be its velocity. In that case, the solution of the stationary problem is known and expresses in cylindrical coordinates $(r, \vartheta, z)$ :

$$
\begin{aligned}
& v_{\vartheta}^{e x}(r)=\frac{C_{1}}{r}+C_{2} r \\
& \tau_{r r}^{e x}(r)=0, \quad \tau_{\vartheta \vartheta}^{e x}(r)=\frac{8 W e \alpha C_{1}^{2}}{r^{4}}, \quad \tau_{r \vartheta}^{e x}(r)=-\frac{2 \alpha C_{1}}{r^{2}}
\end{aligned}
$$

where

$$
C_{1}=\frac{v_{i} r_{i} r_{e}^{2}-v_{e} r_{e} r_{i}^{2}}{r_{e}^{2}-r_{i}^{2}}, \text { and } C_{2}=\frac{v_{e} r_{e}-v_{i} r_{i}}{r_{e}^{2}-r_{i}^{2}}
$$

In the present computations, $r_{i}=15, r_{e}=30, v_{i}=1$ and $v_{e}=0$, while $\Omega=[-15,15] \times[15,25]$ is a rectangular subdomain of the cylindrical section of the flow domain (see Fig. 2). The dimensionless numbers are $W e=2$ and $\alpha=0.99$. The set of equations (2)-(3)-(4) are solved together with boundary conditions $\boldsymbol{v}=\boldsymbol{v}^{e x}$ on $\partial \Omega$ and $\boldsymbol{\tau}=\boldsymbol{\tau}^{e x}$ on $\Gamma_{-}=\{x \in \partial \Omega, \boldsymbol{v} \cdot \boldsymbol{n}<0\}$.

The time-dependent $\theta$-scheme starts with an initial condition at rest and the loop stops when a stationary solution is reached, with $\varepsilon_{G}=10^{-7}$ in (28). Then, the $L^{2}$ norm of the error $e=\boldsymbol{\tau}-\boldsymbol{\tau}^{e x}$ between the approximate FEM solution $\boldsymbol{\tau}$ and the exact one $\tau^{e x}$ is computed. This procedure is repeated on a sequence of uniform meshes with decreasing sizes $h=5 \times 2^{-k}\left(r_{e}-r_{i}\right), k=1, \ldots, 4$. Fig. 3 plots the $L^{2}$ norm of the error versus $h$ in logarithmic scale: observe that the slope is 2. This suggests that the error behaves as $\mathcal{O}\left(h^{2}\right)$, which is optimal for first-order approximation of stresses. Notice that this optimal behavior is better than the $\mathcal{O}\left(h^{3 / 2}\right)$ theoretical bound of the error for the viscoelastic problem [2]: it based on a $\mathcal{O}\left(h^{3 / 2}\right)$ theoretical error bound [23] for the $\mathcal{P}_{1}$ discontinuous 


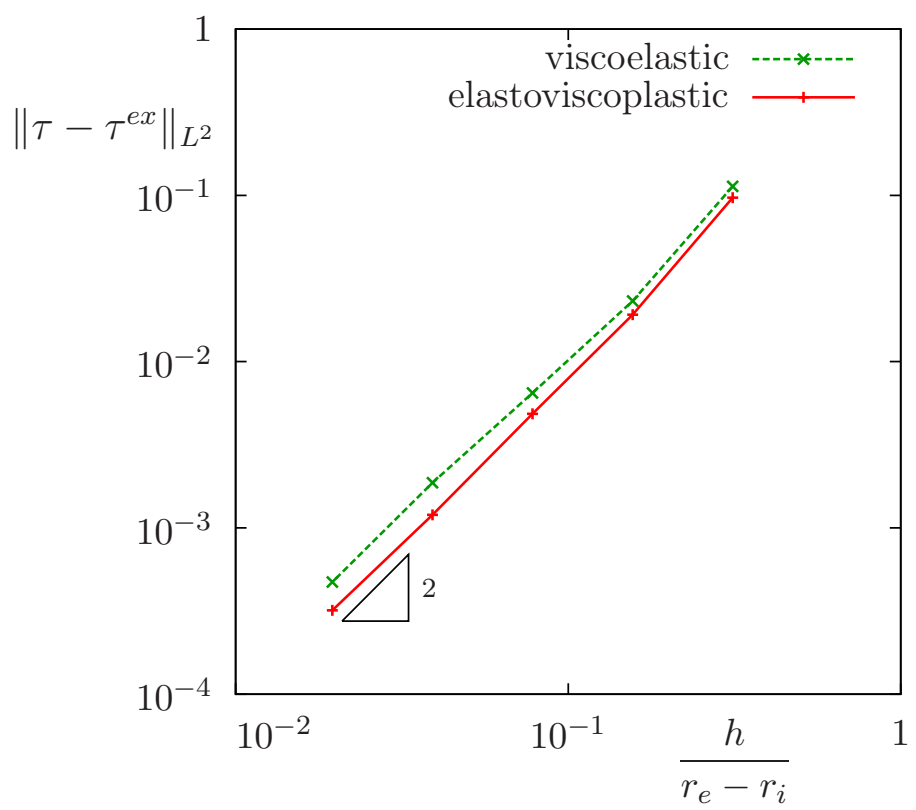

Figure 3: Convergence error versus the mesh size $h$ of the stationary solution: Green dashed line: Oldroyd-B model with $W e=2, \alpha=0.99, a=1$; red solid line: EVP model with $W e=0.1, B i=0.1, \alpha=0.5$.

Galerkin approximation on a pure transport problem. The present numerical results confirm that these theoretical error bounds can be improved for some families of meshes, as pointed out by Richter [36], that showed a $\mathcal{O}\left(h^{2}\right)$ optimal bound for a pure transport problem. This result was recently extended by Cockburn et al. [10], while Peterson [33] showed that the estimate $\mathcal{O}\left(h^{3 / 2}\right)$ is sharp for general families of quasi-uniform meshes.

Let us consider now the full EVP model: the exact solution is not known for the Couette geometry. Nevertheless, thanks to the radial symmetry, it reduces to a one-dimensional problem and has already been solved numerically $[7,9,6]$. A highly accurate numerical solution, associated with 3000 on-dimensional elements in the $\left[r_{i}, r_{e}\right]$ interval, is computed here. This solution is then compared with a two-dimensional computation, in order to validate our computational procedure. The Couette geometry and the computational domain $\Omega$ are unchanged while the material parameters are $W e=0.1, B i=0.1, a=1$ and $\alpha=0.5$. As $B i \neq 0$, the three-fields Stokes problem $(Q 1)$ is non-linear and we use $\varepsilon_{F P}=10^{-8}$ in the stopping criterium (21) of the fixed-point algorithm; as before, the stationary solution is defined with $\varepsilon_{G}=10^{-7}$ in (28). The accurate one-dimensional solution is imposed as a boundary condition on the domain $\Omega$ (see Fig. 2) and we run the bidimensional algorithm for a sequence of uniform meshes with decreasing sizes $h=5 \times 2^{-k}\left(r_{e}-r_{i}\right), k=1, \ldots, 4$. Fig. 3 plots the 
$L^{2}$ norm of the error versus $h$ in logarithmic scale: observe that the slope is 2 . This indicates an optimal rate of convergence of the finite element procedure.

As our numerical procedure is now validated, let us turn to more complex flow simulations, of practical interest and for which the exact solution is not known.

\subsection{Flow arround an obstacle and comparison with experiments}

Flow in a channel around obstacle [45] is a classical setup to probe the rheological of complex fluids, both experimentally and theoretically. In the context of foam rheology, experiments have shown some specificities that cannot be understood if one does not take into account elasticity, viscosity, and plasticity altogether, in a tensorial framework. Furthermore, this experiment avoids some artifacts that can appear in the more classical Couette setup [6].

This flow displays a strong spatial heterogeneity, simultaneous VEP behaviors, a large range of elastic deformations, several elongation and rotation rates, and various relative orientations of the relevant tensors [30]. It enables to follow a bubble at different stages, while it stretches, then while it relaxes: thus, even in a steady flow, transient effects and relaxation times are apparent. It is classically used as a stringent test to discriminate between different models [37].

In addition, experiments with foams or emulsions, especially in 2 dimensions, enable an easy, simultaneous visualization of the micro-structure (bubbles or droplets, which act as tracers of velocity and deformation) and the large scale (global flow heterogeneities). As bubbles slide along the borders with a negligible friction [8], the flow curves are independent of some specific surface rheology that appeared to imply non local effects [20] for some other VEP materials. From a numerical point of view, slip boundary conditions allow to impose a uniform velocity in the $x 1$ direction on the upward boundary $\Gamma_{d}$ of Fig. 1, and a zero stress inbound flux. In the case of a no slip boundary condition, we could use as an inbound condition the solution of the model in a Poiseuille setup, obtained with a high precision with a $1 \mathrm{D}$ algorithm. In [5], we compared the numerical resolution presented here with experimental data (Figs. 4).

The geometry of the problem is detailed in Fig. 1; in particular a slip boundary condition is imposed on the obstacle. A constant velocity $\boldsymbol{v}_{\Gamma}=(1,0)$ is imposed upstream $\Gamma_{d}=\Gamma_{-}$while a Neumann condition is used downstream $\Gamma_{n}$. Since the bidimensional flow is symmetric with respect to the $x_{1}$ axis, only half of the domain is used for the computation and the symmetry condition is similar to the slip condition. Finally, $\Gamma_{s}$ denotes the union of the wall, the obstacle and the symmetry axis boundaries. Upstream, the extra-stress data is $\boldsymbol{\tau}_{\Gamma}=0$. Finally, the initial conditions for $\boldsymbol{\tau}$ and $\boldsymbol{v}$ are taken as zero, since only the stationary solution is of interest for the present flow around an obstacle. The domain has to be long enough both upstream and downstream to ensure that the boundary conditions do not perturb the solution.

Fig. 5 represents how the time-dependent calculations converge towards the 


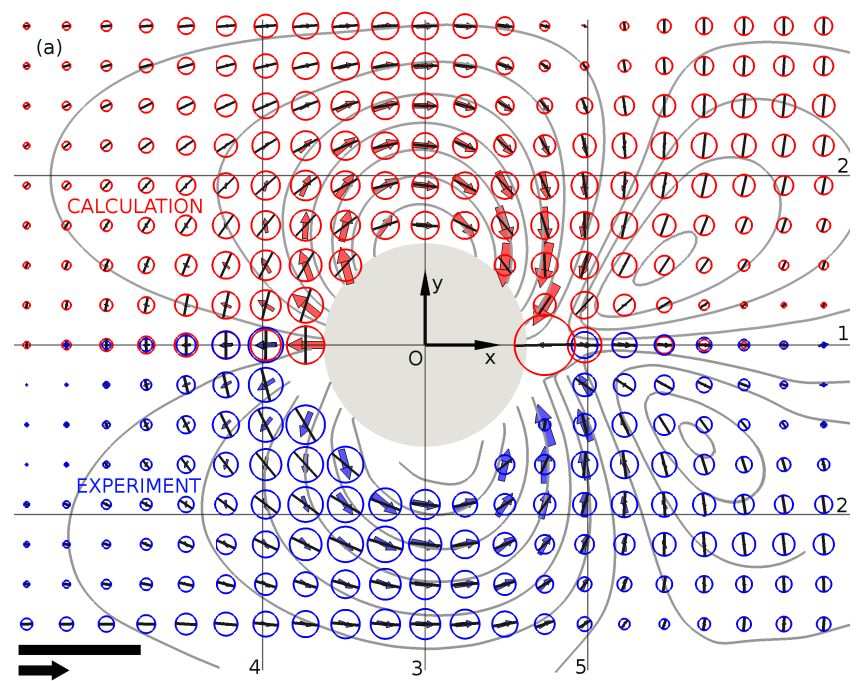

Figure 4: Comparison numerical resolution/experiments, from [5]. Thick colored arrows: velocity field. Circles: elastic deformation tensors; the positive (resp. negative) eigenvalue corresponding to elongation (resp. compression) is represented by a line (resp. not represented). Thin lines: stream lines. Top half (red): present calculation using a continuous model. $\varepsilon_{Y}=0.2, \lambda=0.2 \mathrm{~s}, \eta_{1} / \eta_{2}=0.1, k=0$. Bottom half (blue): experimental data. Scale: bar: 1 (dimensionless) for the elastic eigenvalues (circle diameter); black arrow: entrance velocity $V$.

stationary solution, for the following set of parameters:

$$
W e=0.075, \alpha=0.9, \varepsilon_{Y}=B i W e / 2=0.3, a=1, d t=0.01
$$

and the mesh used for computation is showed in 5 .

The residual term decreases first rapidly, until iterate $n=200$. It then reaches a plateau from $n=300$ to 2800 which corresponds to the advection of initial defects. Note that this advection lasts approximately 3000 iterations, which corresponds to a dimensionless time of 30 as the time step is $d t=0.01$; as the input velocity is unity in dimensionless units, this corresponds to the transport of the defects over the part of the channel downstream of the obstacle. With a shorter downstream domain, the plateau would have been shorter as well. It eventually reaches an asymptotical regime with a constant slope of convergence. Iterations were stopped when the residual term reached $10^{-7}$.

Finally, we check the convergence of the model with respect to mesh size in this complex configuration: Fig. 6 shows the map of the positive part of $\left|\boldsymbol{\tau}_{d}\right|-B i$ : the finer mesh predicts the same yielded area as the coarser one. This is confirmed by cuts of velocity and stress components along the $x_{2}=0$ axis (Fig. 7); in particular, computations with the two meshes predict the same overshoot of the velocity after the obstacle, which is one of the salient features of this setup. 
Such an overshoot, also referred to as "negative wake" in the literature, has already been evidenced, both experimentally and numerically with viscoelastic materials at high velocity (see [5] for a short review) and viscoelastoplastic materials such as Carbopol, with spherical objects settling [35] or flow perpendicular to a disc [24]. These experiments exhibit a strong fore-aft asymmetry with negative wake even in the case of slow flows, which cannot be predicted neither by VE or VP models. As Carbopol is a shear thinning yield stress fluid with elastic properties, it seems that the present approach with the model [43] could be successfully applied to interpret these experiments. 
a) $L^{2}$ norm of the residual (25) vs time iterate.

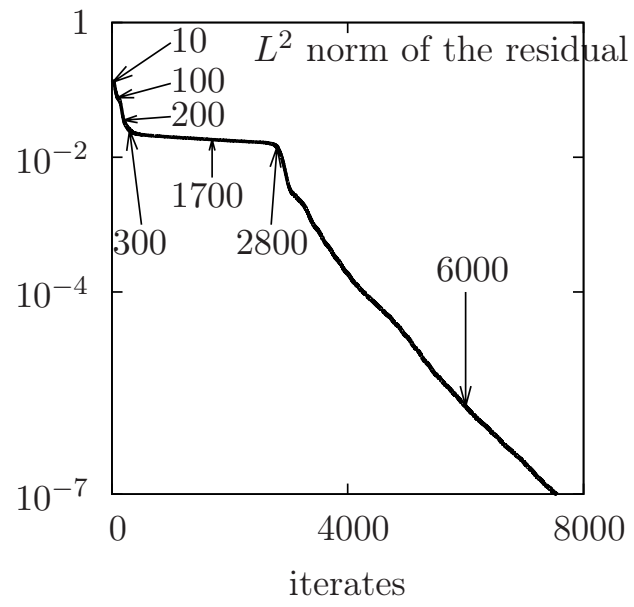

b) iterates $10,100,200$

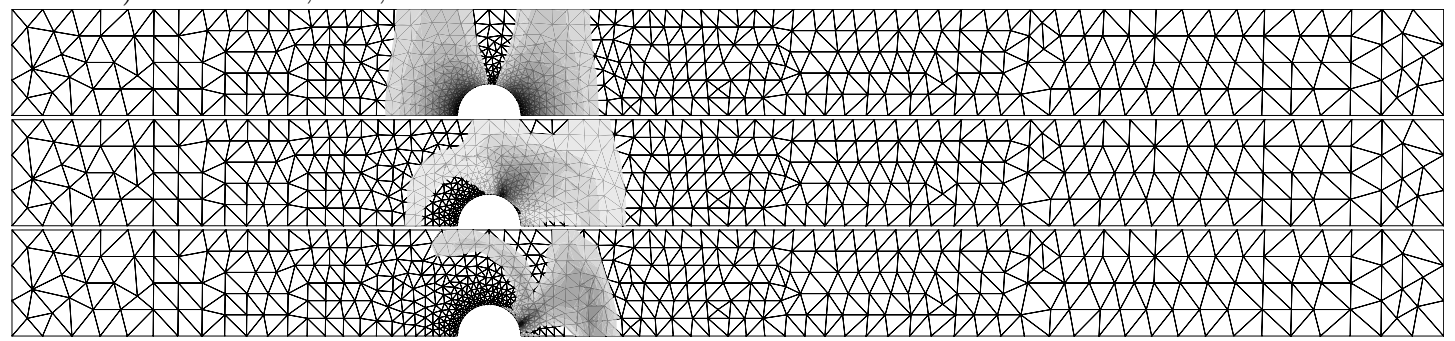

c) iterates $300,1700,2800$

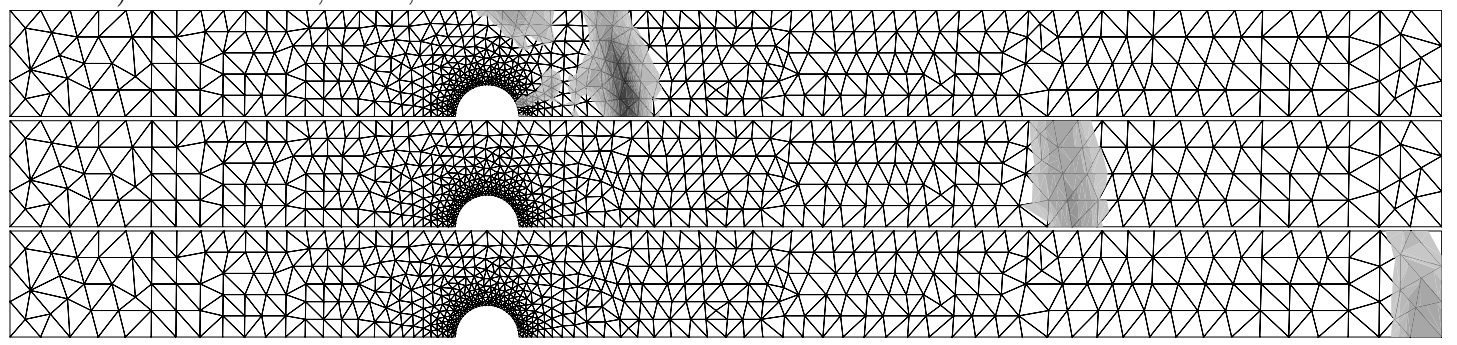

d) iterate 6000

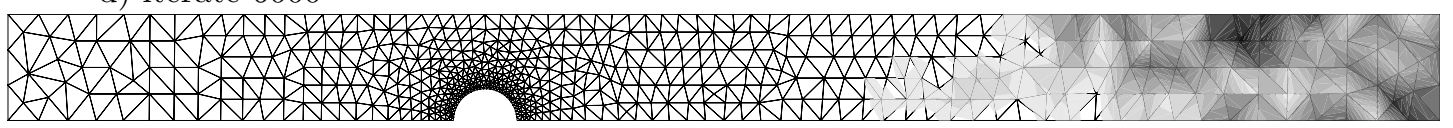

Figure 5: Numerical convergence of the calculation toward stationary solution (see text): a) $L^{2}$ norm over the computational domain $\Omega$ of the residual (25), versus the time iterates; b), c), and d): snapshots of the residual at different time iterates that correspond to the arrows in a); the mesh used for the computation appears in the background. The gray scale is not the same between iterates, as the maximal value of the norm varies over 6 degrees of magnitude; in more details: b) initial phase of fast decrease: different gray scales; c) plateau: same grayscale, from $4 \cdot 10^{-3}$ (light gray) to $2 \cdot 10^{-2}$ (black); d) Steady decreasing phase: from $6.56 \cdot 10^{-8}$ (light gray) to $9.84 \cdot 10^{-7}$ (black). 


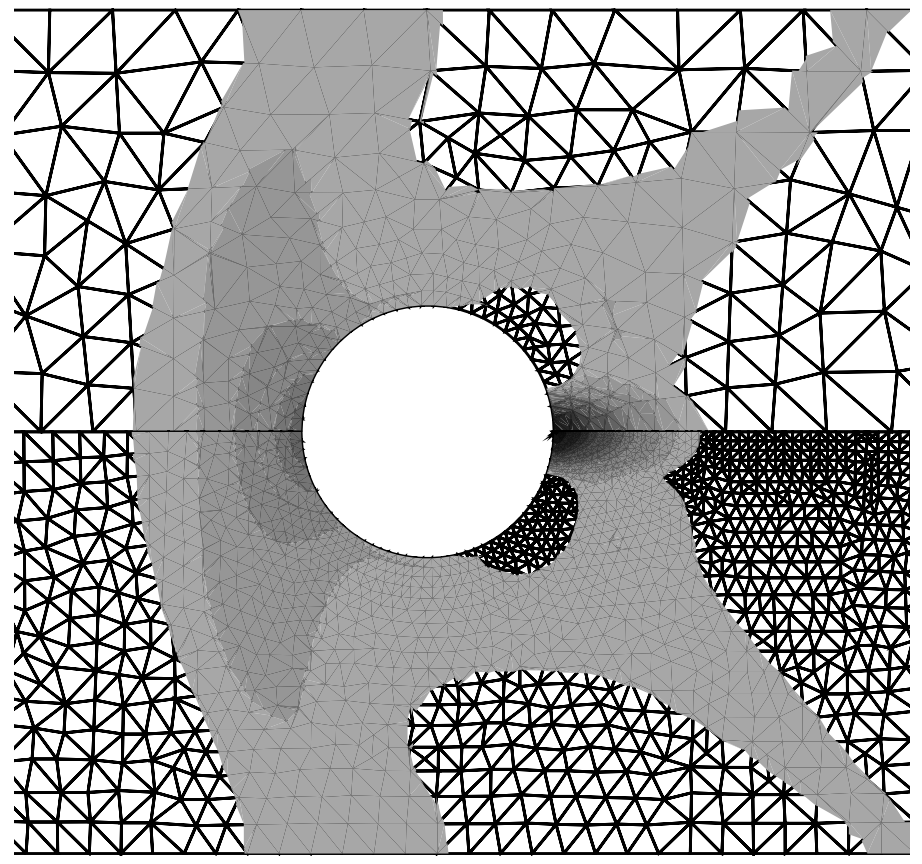

Figure 6: Convergence of the EVP model with the mesh size: positive part of $\left|\boldsymbol{\tau}_{d}\right|-B i$; top: with the mesh used for computations in [5], bottom: with a finer mesh. Same grayscale for both ; values above zero (areas in gray to black) correspond to yielded regions.
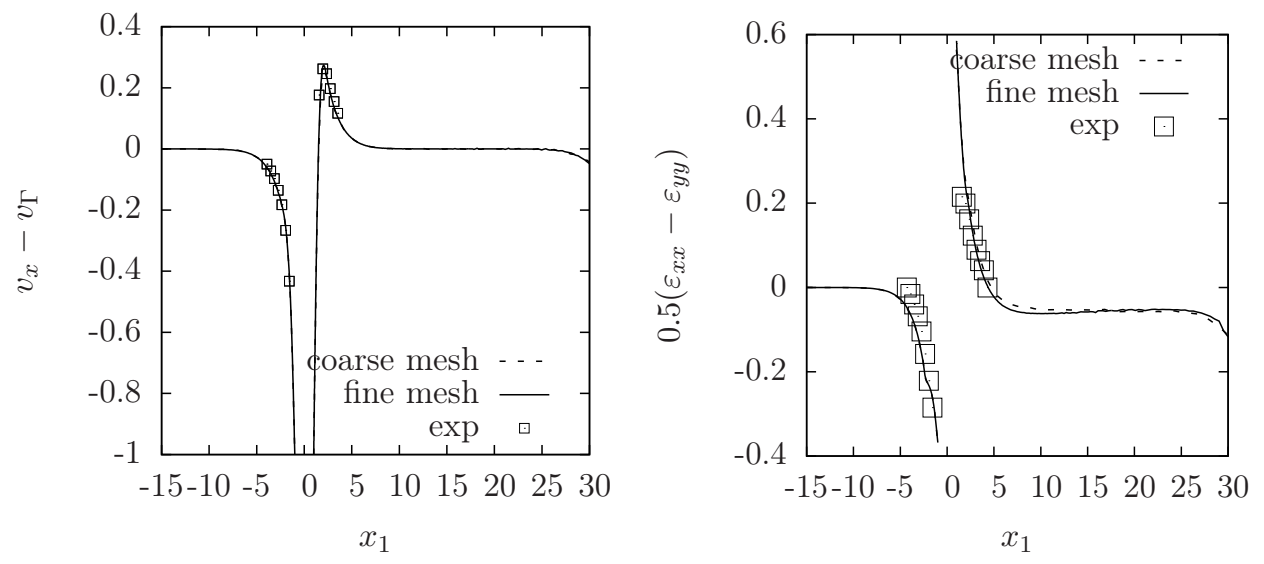

Figure 7: Convergence of the EVP model with the mesh size: cuts of some components of velocity (left) and stress (right) along the $x_{1}$ axis (same data as in [5]). 


\section{Conclusion}

An efficient time-dependent decoupled approach, called a $\theta$-scheme, was presented in this paper. This approach allows to decouple the two main difficulties of EVP problems: the viscoplasticity and the viscoelasticity, resulting in two subproblems. The first subproblem, containing the nonlinear terms due to viscoplasticity is solved by a fixed point algorithm. The second subproblem, related to viscoelasticity, reduces to a tensorial linear transport problem, involving the material derivative. This splitting of the two difficulties leads to an efficient solver. This efficiency is confirmed by the numerical results presented in this paper: an optimal convergence rate with respect to the space discretization and a fast and robust convergence to a stationary solution. Finally, the solution of the EVP model compares well with experimental measurements, leading to both qualitative and quantitative agreement for liquid foams in a complex flow problem, the flow around an obstacle. This model allows realistic predictions of both the velocity field and the elastic stress. Future works will explore flows of liquid foams for some tridimensional geometries where experimental data are available and will also compare the model to flows of others soft glassy materials such as Carbopol solutions. The present splitting algorithm could also be extended to solve the case of non-constant viscosity and relaxation time: see e.g. [13] for applications to thixotropic elastoviscoplastic fluids and [19] for non-isothermal and thixotropic viscoplastic fluids.

[1] E. Bansch and K. Deckelnick. Optimal Error Estimates for the NavierStokes Equations with Slip Boundary Condition. M2AN Math. Model Numer. Anal., 33:923-938, 1999.

[2] J. Baranger and D. Sandri. Finite element approximation of viscoelastic fluid flow: existence of approximate solutions and error bounds. part i. discontinuous constraints. Numer. Math., 63:13-27, 1992.

[3] S. Bénito, C.-H. Brunneau, T. Colin, C. Gay, and F. Molino. An elastovisco-plastic model for immortal foams or emulsions. Eur. Phys. J. E, 25:225-251, 2008.

[4] E. C. Bingham. Fluidity and plasticity. Mc Graw-Hill, 1922.

[5] I. Cheddadi, P. Saramito, B. Dollet, C. Raufaste, and F. Graner. Understanding and predicting viscous, elastic, plastic flows. Eur. Phys. J. E. Soft matter, 34(1):11001, 2011.

[6] I. Cheddadi, P. Saramito, and F. Graner. Stationary Couette flows of elastoviscoplastic fluids are non-unique. J. Rheol., 56(1):213-239, 2012.

[7] I. Cheddadi, P. Saramito, C. Raufaste, P. Marmottant, and F. Graner. Numerical modelling of foam Couette flows. Eur. Phys. J. E, 27(2):123$133,2008$. 
[8] I. Cheddadi, P. Saramito, C. Raufaste, P. Marmottant, and F. Graner. Numerical modelling of foam Couette flows. Eur. Phys. J. E, 27:123-133, 2008.

[9] I. Cheddadi, P. Saramito, C. Raufaste, P. Marmottant, and F. Graner. Erratum to numerical modelling of foam Couette flows. Eur. Phys. J. E, 28:479-480, 2009.

[10] B. Cockburn, B. Dong, J. Guzmán, and J. Qian. Optimal convergence of the original DG method on special meshes for variable transport velocity. SIAM J. Numer. Anal., 48(1):133-146, 2010.

[11] P. Coussot. Rheometry of Pastes, Suspensions and Granular Materials. Wiley, 2005.

[12] B. Dollet and F. Graner. Two-dimensional flow of foam around a circular obstacle: local measurements of elasticity, plasticity and flow. J. Fluid Mech., 585:181-211, 2007.

[13] C. Fonseca, S. Frey, M. F. Naccache, and P. R. de Souza Mendes. Flow of an elasto-viscoplastic thixotropic fluid past a confined cylinder. J. Non-Newt. Fluid Mech., 193:80-88, 2013.

[14] M. Fortin and A. Fortin. A new approach for the FEM simulation of viscoelastic flows. J. Non-Newtonian Fluid Mech., 32(3):295-310, 1989.

[15] K. O. Friedrichs. Symmetric positive linear differential equations. Comm. Pure Appl. Math., 11:333-418, 1958.

[16] V. Giraut and P.-A. Raviart. Finite Element Approximation of the NavierStokes Equations. Springer-Verlag Heidelberg, 1979.

[17] R. Glowinski and P. Le Tallec. Augmented Lagrangian and operator splitting methods in nonlinear mechanics. SIAM, Philadelphia, USA, 1989.

[18] R. Glowinski, B. Mantel, and J. Périaux. Numerical solution of the time dependent Navier-Stokes equations for incompressible viscous fluids by finite element and alternating direction methods, pages 309-336. Academic Press, London and New York, 1982.

[19] R. Glowinski and A. Wachs. On the numerical simulation of viscoplastic fluid flow. In P. G. Ciarlet and J.-L. Lions, editors, Handbook of numerical analysis. Volume 16. Numerical methods for non-Newtonian fluids, chapter 6, pages 483-717. Elsevier, 2011.

[20] J. Goyon, A. Colin, G. Ovarlez, A. Ajdari, and L. Bocquet. Spatial cooperativity in soft glassy flows. Nature, 454:84-87, 2008.

[21] W. H. Herschel and T. Bulkley. Measurement of consistency as applied to rubber-benzene solutions. Am. Soc. Test Proc., 26:621-633, 1926. 
[22] E. Janiaud, D. Weaire, and S. Hutzler. Two-dimensional foam rheology with viscous drag. Phys. Rev. Lett., 97:038302, 2006.

[23] C. Johnson and J. Pitkäranta. An analysis of the discontinuous Galerkin method for a scalar hyperbolic equation. Math. Comp., 46(173):1-26, 1986.

[24] L. Jossic, F. Ahonguio, and A. Magnin. Flow of a yield stress fluid perpendicular to a disc. J. Non-Newt. Fluid Mech., 191:14-24, 2013.

[25] G. Katgert, M. E. Möbius, and M. van Hecke. Rate dependence and role of disorder in linearly sheared two-dimensional foams. Phys. Rev. Lett., 101:058301, 2008.

[26] P. Lesaint and P. A. Raviart. Finite element collocation methods for first order systems. Math. Comput., 33(147):891-918, 1979.

[27] L.-H. Luu and Y. Forterre. Drop impact of yield-stress fluids. J. Fluid Mech., 632:301-327, 2009.

[28] P. Marmottant, A. Mgharbel, J. Käfer, B. Audren, J. P. Rieu, J.-C. Vial, B. van der Sanden, A. F. M. Marée, F. Graner, and H. Delanoë-Ayari. The role of fluctuations and stress on the effective viscosity of cell aggregates. Proc. Natl. Acad. Soc., 106:17271, 2009.

[29] P. Marmottant, C. Raufaste, and F. Graner. Discrete rearranging disordered patterns, part II: 2D plasticity, elasticity and flow of a foam. Eur. Phys. J. E, 25:371-384, 2008.

[30] P. Marmottant, C. Raufaste, and F. Graner. Discrete rearranging disordered patterns, part II: 2D plasticity, elasticity and flow of a foam. Eur. Phys. J. E, 25:371-384, 2008.

[31] J. G. Oldroyd. A rational formulation of the equations of plastic flow for a Bingham fluid. Proc. Cambridge Philos. Soc., 43:100-105, 1947.

[32] J. G. Oldroyd. On the formulation of rheological equations of states. Proc. Roy. Soc. London, A200:523-541, 1950.

[33] T. E. Peterson. A note on the convergence of the discontinuous Galerkin method for a scalar hyperbolic equation. SIAM J. Numer. Anal., 28(1):133$140,1991$.

[34] L. Preziosi, D. Ambrosi, and C. Verdier. An elasto-visco-plastic model of cell agregates. J. Theor. Biol., 262:35-47, 2010.

[35] A. M. V. Putz, T. I. Burghelea, A. Frigaard, and D. M. Martinez. Settling of an isolated spherical particle in a yield stress shear thinning fluid. Phys. Fluids, 20:033102, 2008.

[36] G. R. Richter. An optimal-order error estimate for the discontinuous galerkin method. Math. Comput., 50(181):75-88, 1988. 
[37] N. Roquet and P. Saramito. An adaptive finite element method for Bingham fluid flows around a cylinder. Comput. Appl. Meth. Mech. Engrg., 192:3317-3341, 2003.

[38] P. Saramito. Numerical simulation of viscoelastic fluid flows using incompressible finite element method and a $\theta$-method. M2AN, 28(1):1-35, 1994.

[39] P. Saramito. Operator splitting for viscoelastic fluid with a differential constitutive law. C. R. Acad. Sci. Paris, ser. II, 319(3):267-270, 1994.

[40] P. Saramito. Efficient simulation of nonlinear viscoelastic fluid flows. J. Non Newtonian Fluid Mech., 60:199-223, 1995.

[41] P. Saramito. Operator splitting in viscoelasticity. Élasticité, Viscoélasticité et Contrôle Optimal, Lyon, décembre 1995, ESAIM: Proceedings, 2:275281, 1997.

[42] P. Saramito. A new constitutive equation for elastoviscoplastic fluid flows. J. Non-Newtonian Fluid Mech., 145:1-14, 2007.

[43] P. Saramito. A new elastoviscoplastic model based on the Herschel-Bulkley viscoplasticity. J. Non Newtonian Fluid Mech., 158:154-161, 2009.

[44] P. Saramito and J.-M. Piau. Flow characteristics of viscoelastic fluids in an abrupt contraction by using numerical modeling. J. Non Newtonian Fluid Mech., 52:263-288, 1994.

[45] G. G. Stokes. On the effect of the inertial friction of fluids on the motion of pendulums. Cambr. Philos. Soc. Trans., 9:8-106, 1851.

[46] R. Verfürth. Finite element approximation of steady Navier-Stokes equations with mixed boundary conditions. M2AN Math. Model Numer. Anal., 19:461-475, 1985.

[47] R. Verfürth. Finite element approximation of incompressible Navier-Stokes equations with slip boundary conditions I. Num. Math., 50:697-721, 1987. 